\title{
Benefits of Force Limited Vibration Testing
}

\author{
Mark E. McNelis ${ }^{\dagger}$ and \\ NASA John Glenn Research Center, Cleveland, Ohio USA
}

Terry D. Scharton ${ }^{\dagger}$

Jet Propulsion Laboratory, Pasadena, California USA

(Received 20 November 1999; revised 21 March 2000; accepted 6 July 2000)

\begin{abstract}
Force limited random vibration testing is used at NASA John Glenn Research Center (formerly NASA Lewis Research Center) for the purposes of qualifying hardware for flight. The benefit of force limited testing is that it limits overtesting of flight hardware, by controlling input force and acceleration from the shaker (dual control) to the test article. The purpose of force limiting is to replicate the test article resonant response for the flight mounting condition. The force limited testing technology has been implemented at the Jet Propulsion Laboratory for the past 10 years on various spacecraft testing programs. For example, the Cassini mission to Saturn utilised force limited vibration testing extensively for both the instrument and spacecraft system level vibration testing. ${ }^{1}$ NASA John Glenn Research Center is responsible for microgravity combustion and fluid science research on the Shuttle and the International Space Station. Qualification testing of delicate and vibration sensitive science instrumentation is particularly challenging to qualify successfully for flight. In order to facilitate the testing process, force limiting has been implemented. This paper addresses recent flight camera testing (qualification random vibration and strength testing) for the Combustion Module-2 mission and the semi-empirical method of specifying force limits.
\end{abstract}

${ }^{\dagger}$ Member of the International Institute of Acoustics and Vibration (IIAV)

\section{INTRODUCTION}

The force limited technique of controlling both the input acceleration and force in vibration testing (dual control) has been implemented to reduce overtesting of aerospace hardware. The major cause of overtesting in vibration tests is due to the impedance mismatch between the flight boundary condition and the test configuration. The consequence of the impedance mismatch is that the maximum shaker force spectrum during a vibration test is typically higher, sometimes by a factor of a thousand, than the maximum interface force spectrum during flight. ${ }^{2}$ The problem arises because the flight interface acceleration spectrum always has notches at the fixed base resonance frequencies of the test item, and test input acceleration spectrum is, at best, a frequency envelope of the actual flight environment, the details of which are seldom known a priori.

The application of force limiting has several benefits over traditional acceleration controlled vibration testing. Force limiting eliminates the interface impedance mismatch thereby limiting the test item response at resonance. Force limiting also provides a means to measure and limit the acceleration of the structure's center of gravity. Aerospace structural design load factors are defined at the center of gravity of the structure; the design load factors can be used as a constraint for the definition of maximum input force. In this way, force limiting can be implemented as a strength test.

\section{FORCE LIMITING THEORY AND APPLICATION}

NASA Glenn Research Center's Structural Dynamics Laboratory performs structural dynamic testing to qualify hardware for flight. The test results for the random vibration testing of the Combustion Module-2 (CM-2) Xybion camera package and the implementation of the force limiting technology are presented.

The advent of commercially available, economically priced, and miniaturised three-axis piezoelectric force gages has facilitated the use of force limiting for practical application in the vibration test laboratory. A monograph on force limiting ${ }^{3}$ contains a theoretical discussion of force limiting and some case histories. A NASA Technical Handbook ${ }^{4}$ offers practical guidance on conducting force limited vibration tests including the derivation of limit criteria and implementation considerations such as force gage preload, calibration, fixturing, and the control strategy. The force gages (washers) are installed in series between the test article and shaker table interface. Control accelerometers are installed in parallel with the test article. The control system adjusts the input force and acceleration signals using a dual control algorithm, which increases the shaker drive signal at each frequency so that either the acceleration or force is at its specified limit, but neither exceeds its limit.

For the CM-2 Xybion camera package vibration testing, the semi-empirical method ${ }^{3-5}$ was used to define the force limits. For random vibration, the semi-empirical method may be stated as:

$$
\begin{array}{ll}
S_{F F}=C^{2} M_{0}^{2} S_{A A} & f<f_{0} ; \\
S_{F F}=C^{2} M_{0}^{2} S_{A A} /\left(f / f_{0}\right)^{2} & f>f_{0},
\end{array}
$$

where $S_{F F}$ is the force limit spectral density, $C$ is a constant which depends on the configuration and the limit criteria, $M_{0}$ is the total mass of the test item, $S_{A A}$ is the acceleration control spectral density, $f$ is the frequency in hertz, and $f_{0}$ is the fundamental resonance frequency (in hertz) of the test article. 